\title{
Completed suicide in an autopsy-confirmed case of early onset Alzheimer's disease
}

\author{
Jennifer Wiener Hartzell*,1,2, Richard Geary ${ }^{3}$, Kymberly Gyure ${ }^{4,5,6}$, Venkata Ravi Chivukula \\ \& Marc W Haut ${ }^{5,7,8}$ \\ ${ }^{1}$ Department of Supportive Oncology, Levine Cancer Institute, Carolinas HealthCare System, NC 28202, USA \\ ${ }^{2}$ Department of Neuropsychology \& Psychology, Carolinas Rehabilitation, Carolinas HealthCare System, NC 28203, USA \\ ${ }^{3}$ Department of Psychiatry, Overlake Medical Center, WA 98004, USA \\ ${ }^{4}$ Department of Pathology, West Virginia University School of Medicine, WV 26506, USA \\ ${ }^{5}$ Department of Neurology, West Virginia University School of Medicine, WV 26506, USA \\ ${ }^{6}$ Department of Neurosurgery, West Virginia University School of Medicine, WV 26506, USA \\ ${ }^{7}$ Department of Behavioral Medicine \& Psychiatry, West Virginia University School of Medicine, WV 26505, USA \\ ${ }^{8}$ Department of Radiology, West Virginia University School of Medicine, WV 26506, USA \\ *Author for correspondence: jennifer.hartzell@carolinashealthcare.org
}

We report a case of a 57-year-old male with clinically diagnosed and autopsy-confirmed early onset Alzheimer's disease who completed suicide by gunshot wound to the chest. This case has several unique aspects that have not been discussed in previous case reports of completed suicide in Alzheimer's disease. In particular, our patient's death was highly planned with successful compensation for his cognitive deficits. After all firearms had been removed from the home as a safety precaution, he obtained a new weapon, hid it and left himself cues to find and use it. The case is discussed in the context of literature differentiating the neural circuitry propagating impulsive versus planned suicidal acts.

First draft submitted: 3 October 2017; Accepted for publication: 18 December 2017; Published online: 10 January 2018

Keywords: Alzheimer's disease $\bullet$ early onset $\bullet$ suicide

We present a case of a 57-year-old, right-handed Caucasian male clinically diagnosed with early onset Alzheimer's disease (AD), which was confirmed on autopsy after he completed suicide in October 2014 by a self-inflicted gunshot wound to the chest. He originally presented to our medical school-based Memory Disorder Clinic in January 2013 with complaints of forgetfulness over the last 5 years. He was working full-time and performed all activities of daily living independently. In addition to slowly progressive memory concerns, he reported anhedonia, decreased energy, restless sleep, decreased appetite and unintentional weight loss, but did not endorse depressed mood. He had no suicidal ideation at initial presentation. His affect was noted to be constricted.

Medical history was otherwise notable for gallbladder disease and mild sleep apnea. He had no history of head injury or concussive events. Computed tomography of the brain in 2011 was negative. Daily medications included baby aspirin and vitamin $\mathrm{D}$. He did not use a continuous positive airway pressure ventilator. Substance use history was unremarkable.

A few years prior to presenting for treatment in our setting, he was prescribed escitalopram for symptoms of anxiety and depression, but discontinued on his own after 2 weeks, and his symptoms resolved over time. He had also participated in marital counseling for a few months over the summer prior to his intake. Otherwise, psychiatric history was unremarkable and he had no history of self-harm or suicide attempts.

Family history, however, was quite relevant. The patient's only brother died from Wilson's disease in 1993. His father killed himself by gunshot in 1994, 1 year after receiving a diagnosis of terminal lung cancer and losing his son. He was 74 years old at the time, but did not have any reported memory problems. His mother was still alive, but in an assisted-living facility for an unspecified dementia syndrome. Her memory problems began at the age of 85. A maternal uncle was also reported to have late-life memory problems.

At intake, he scored 22/30 on the Mini Mental Status Examination (MMSE) [1]. Laboratory values, including thyroid stimulating hormone, rapid plasma regain, B12 and folate, were normal. Serum ceruloplasmin was negative 


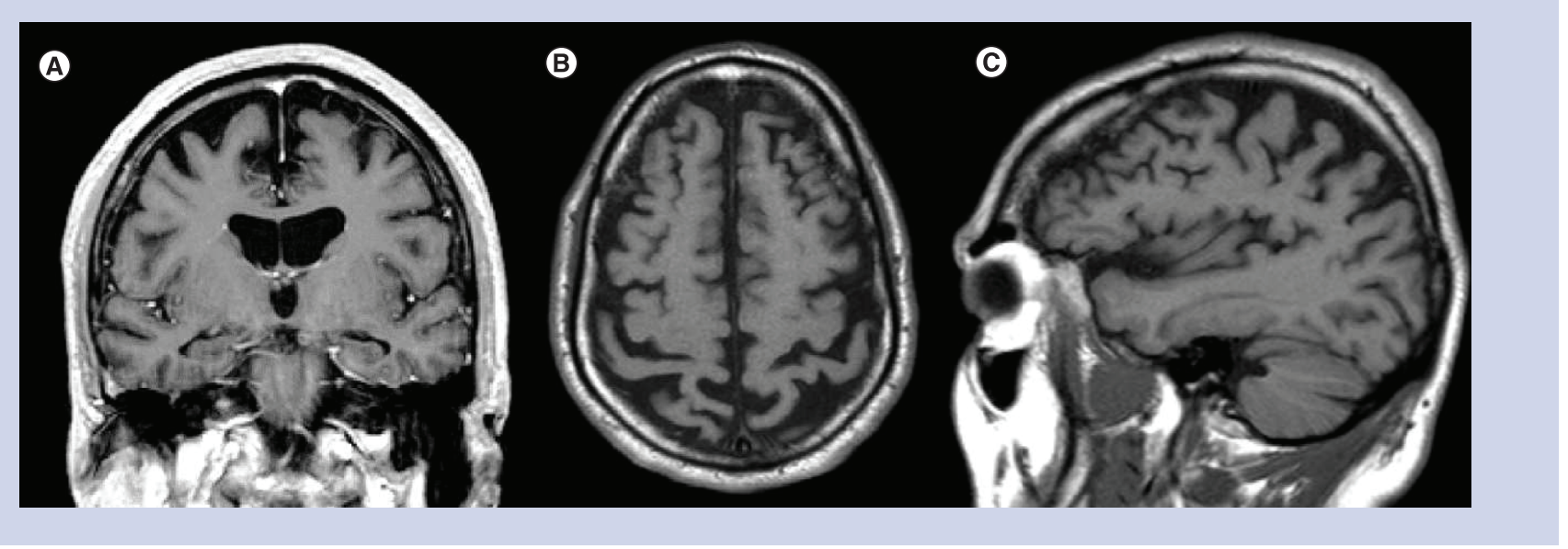

Figure 1. MRI of the brain.(A) Coronal T1-weighted image post contrast. (B) Axial T1-weighted image. (C) Sagittal T1-weighted image.

for Wilson's disease. Bupropion (100 mg) was prescribed and he was referred for neuropsychological evaluation, MRI and EEG.

One month later (February 2013), neuropsychological evaluation revealed a pattern of cognitive deficits suspicious for early onset $\mathrm{AD}$ with impairments in memory encoding and consolidation, verbal fluency, visual construction, attention, verbal reasoning and cognitive flexibility. He scored 18/30 on the MMSE at that point and was not fully oriented to time. He had functional deficits related to making change from a simulated purchase and writing a check, which is of note considering that he had a Bachelor's degree in finance and worked as a banking executive. He was informed of the results and preliminary diagnosis at a feedback appointment with the neuropsychologist 2 weeks later and appeared to understand and accept the results, but demonstrated restricted affect and reduced insight.

MRI of the brain from April 2013 revealed generalized atrophy considered to be advanced for his age. As shown in Figure 1A, there is hippocampal atrophy evident on the coronal T1-weighted image, but the atrophy of the hippocampus is not out of proportion to the atrophy elsewhere in the brain. Examination of the axial and sagittal T1 images (Figure 1B \& C) suggests there may be more atrophy in the parietal cortex than the other lobes, as has been reported in the setting of early onset AD [2,3]. His EEG was normal.

When our patient returned to the Memory Disorder Clinic for follow-up in March 2013, he reported improved mood, energy and sleep with weight gain. He did not endorse hopelessness or suicidal ideation. His wife noted improved memory. Bupropion was increased to $300 \mathrm{mg}$. In June 2013, he reported continued benefit from bupropion and no overt depression. He was formally diagnosed with early onset $\mathrm{AD}$ after seeking a second opinion at another academic medical center. He was considering quitting his job and applying for disability based on his diagnosis. Donepezil $(10 \mathrm{mg})$ was prescribed and he requested information about genetic testing for AD.

By August 2013, he retired and applied for disability. His mood had declined. Bupropion was maintained at $300 \mathrm{mg}$. He reported experiencing a single visual hallucination of a cat, but was not distressed by this occurrence. He returned to clinic in December 2013, at which time his depression had worsened with disturbances in sleep and appetite. He also reported increased anxiety. Prior medications were maintained and he was started on memantine $(28 \mathrm{mg})$. He returned in January 2014 with improved mood, sleep, appetite and anxiety. He returned in May 2014 and was doing well, tolerating bupropion, donepezil and memantine.

In July 2014, he was seen for a repeat neuropsychological evaluation to monitor cognitive progression. $\mathrm{He}$ reported no depression or suicidal ideation. Family confirmed that he had no access to guns in the home, as they had been removed. He was no longer managing his finances or medications. He continued to drive short distances on his own, but his wife indicated that she was no longer comfortable as a passenger. He characterized his retirement as being 'let go', which was not accurate, and was now receiving disability. His MMSE was 16/30 and Geriatric Depression Scale 15-item (GDS-15) was 3/15, indicating minimal depression. The results of the neuropsychological re-evaluation revealed progressive decline across cognitive domains. He performed at the first percentile across measures of verbal and nonverbal memory, the second percentile on a confrontation naming task 


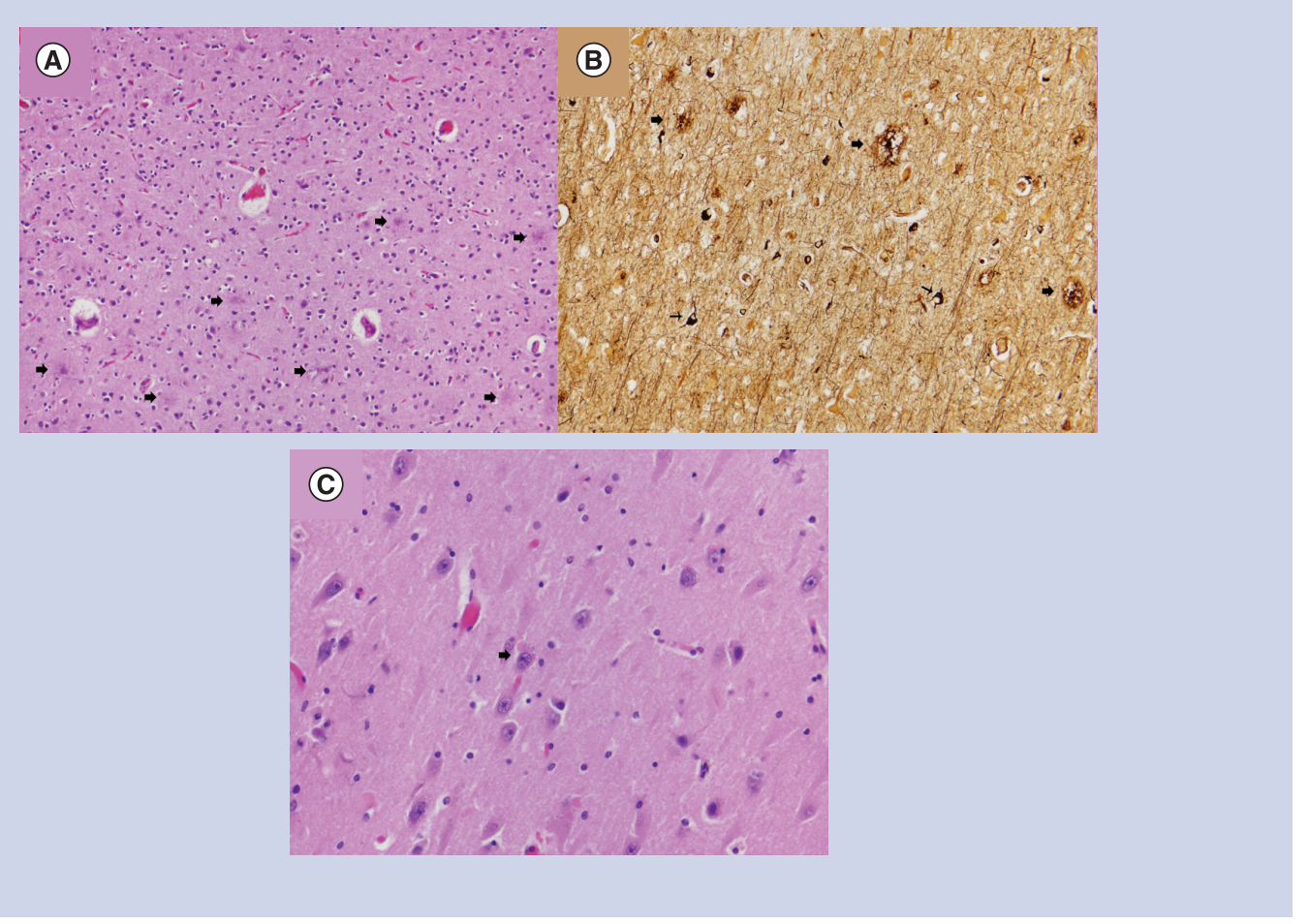

Figure 2. Neuropathology slides.(A) Neuritic amyloid plaques (arrows) were readily identified in all neocortical regions examined (hematoxylin-eosin, original magnification $200 \times$ ). (B) Neuritic plaques (thick arrows) and neurofibrillary tangles (thin arrows) were highlighted with a Bielschowsky silver stain (original magnification 200x). (C) Hippocampal pyramidal neurons exhibited granulovacuolar degeneration (arrow) and contained Hirano bodies (hematoxylin-eosin, original magnification $400 \times$ ).

and the 16th percentile on an abstract reasoning task. He was unable to complete a visual sequencing task and could not comprehend the instructions for a measure of cognitive flexibility. His overall cognitive pattern and rate of progression were determined to be consistent with a moderately advanced early onset AD.

He returned to the Memory Disorder Clinic in October 2014. His depression had recurred, yet he only reported mild symptoms on the GDS-15 (6/15). He expressed 'slipping' cognitively. He found enjoyment in mowing the lawn and spending time with his grandson. He attributed his depressed mood to concern over his mother's health, which had declined. He made no mention of suicidal ideation. No medication changes were made.

He presented to the emergency department at the end of October 2014 with a gunshot wound to his chest, which was ruled suicide. He died in the operating room. At autopsy, examination of the brain conducted by a board-certified neuropathologist revealed that the brain weighed $1440 \mathrm{~g}$ and exhibited mild, diffuse narrowing of the gyri with deepening of the sulci. Coronal sections demonstrated symmetric dilatation of the lateral ventricular system, including the temporal horns. Hematoxylin-eosin-stained microscopic sections including those from the medulla, pons, midbrain, cerebellar vermis, cerebellar dentate, bilateral hippocampi, basal ganglia, thalamus, middle frontal gyrus, inferior parietal lobule, occipital lobe, cingulate gyrus and superior and middle temporal gyri were evaluated. Selected sections were additionally stained with a Bielschowsky silver stain. Frequent neuritic amyloid plaques were identified in the hippocampi, the adjacent entorhinal cortices and all neocortical regions examined, including the primary visual cortex of the occipital lobe (Figure 2A). Frequent neurofibrillary tangles were also present in a similar distribution (Figure 2B). In addition, there was granulovacuolar degeneration of and Hirano bodies present within hippocampal pyramidal neurons (Figure 2C). No brainstem or cortical Lewy bodies or other abnormal inclusions were identified. Using modified National Institute on Aging-Alzheimer's Association guidelines for the neuropathologic assessment of $\mathrm{AD}$, the degree of $\mathrm{AD}$ neuropathologic change was considered sufficient to explain this patient's dementia [4]. Staging of the neuropathology of the disease was not completed at the time of the autopsy. 


\section{Background}

Prevalence rates of suicide in $\mathrm{AD}$ vary, with reports ranging from less than $1 \%$ to as high as $11 \%$ [5-7]. Some assert that dementia and $\mathrm{AD}$ specifically confer a lower risk of suicidal behavior in light of cognitive compromise [8,9], while others contend there is at least a mild-moderate risk [10-13]. A prospective study out of Denmark examining all cases of hospital-diagnosed dementia based on national registry data over an 11-year span revealed an elevated risk of completed suicide in adults over 50 following dementia diagnoses. Risk was highest in the first 6 months following diagnosis, but the occurrence of suicide more than a decade later was also high. Furthermore, the association between dementia and suicide risk remained significant even after controlling for current and past mood disorders. The authors comment on how surprising it is that so many cognitively impaired individuals, by nature of their disease, are capable of planning and executing a successful suicide [14]. We agree and believe that our case, relative to previous case reports, uniquely highlights just how surprising, yet realistic and possible, suicide is in the context of progressive cognitive impairment.

There are a handful of previously published case reports of completed suicide in $\mathrm{AD}$. Common features of these cases include relatively preserved insight and higher educational attainment. Early age of onset of the disease, defined as clinical onset before age 65, is present in three of eight cases of completed suicide [15].

Rohde, Peskind and Raskind [16] reported two cases of completed suicide from a university-affiliated Alzheimer's program. Both cases were clinically diagnosed with probable $\mathrm{AD}$ and enrolled in clinical trials. The first case was a 54-year-old woman who visited Dr Jack Kevorkian for physician-assisted suicide. AD was confirmed at autopsy. The second case was an 80-year-old male who died of a self-inflicted gunshot wound. Autopsy was not performed. Both individuals were highly educated professionals in the early stages of the disease with intact insight into their prognoses.

A second set of completed suicide cases came from a Phase III clinical trial with eptastigmine, a cholinesterase inhibitor [17]. The first case was a 57 -year-old man who died of a self-inflicted gunshot wound, while the second was a 73-year-old divorced male who jumped to his death from a 19th-floor window. Both individuals were well-educated professionals who met clinical diagnostic criteria for probable AD. Pathology was not discussed.

A third set of cases revealed preserved insight in highly educated males who were more advanced in age at the time of clinical diagnosis [18]. The first was an 87-year-old who attempted suicide twice by wrist slashing, but ultimately died of respiratory failure at the age of 91 . The second case was an 82-year-old who died of a self-inflicted gunshot wound to the head. In both cases, $\mathrm{AD}$ was confirmed at autopsy.

There is a single case report of presumed AD in a 58-year-old, well-educated male who died by gas inhalation, but his disease was not confirmed by autopsy [19]. At last, there is one additional case in the literature that was part of a 1986 murder-suicide in which a 67-year-old man died of a self-inflicted gunshot wound to the head and autopsy revealed plaques and tangles in the frontal lobes and left temporal lobe. Retrospectively, it was reported that he demonstrated cognitive and behavioral changes in the 2 years preceding his death; however, he had not been clinically diagnosed with dementia or $\mathrm{AD}$ while living [20].

\section{Discussion}

While our case of completed suicide in the setting of early onset $\mathrm{AD}$ has similarities to the cases discussed above, such as being a well-educated male with relative insight into his prognosis, there are unique aspects to this case that have not been reported previously, which we will highlight.

First, our patient's death was a highly planned event. Following his suicide, his wife reported that he had purchased a new gun, unknown to her, after all firearms had been removed from the home as instructed by his providers. Generalizing from the literature examining planned versus impulsive suicides, the behavior eventually leading to our patient's death is consistent with the neuropathology of his disease. Specifically, as we detail below, planned or premeditated suicides are associated with alterations in cortical processes, whereas impulsive suicidal behavior reflects alterations in subcortical processes [21]. Thus, our patient's planned act was more likely to be related to cortical brain dysfunction, as is seen in $\mathrm{AD}$.

Second, he appears to have successfully compensated for his cognitive deficits by leaving himself cues. After his death, we learned that he hid a newly purchased gun in a lockbox in the garage, tied a ribbon around the lockbox, marked the key to the lockbox and put the key on his keychain. Thus, despite his disease being moderately advanced at the time of his death, he maintained a level of awareness of his prognosis and sufficient executive functions at this point in the course of his disease to design and implement a suicide plan. His ability to accomplish this act may indicate a higher level of cognitive reserve (CR). The concept of CR posits that individual differences in 
factors such as educational and occupational attainment offer neural resiliency and the ability to compensate for pathologic brain changes, such as those occurring in $\mathrm{AD}$ [22]. Considering our patient's status as a college-educated bank executive, high CR may have moderated the relationship between his pathology and his behavior.

Third, our patient never presented with the agitated or hopeless presentations of major depression that have traditionally been perceived as 'risk factors' for suicidal behavior [23-25]. Rather, his depression came and went. When it was present, his depression was generally characterized by apathy, anhedonia and constricted affect instead of frank dysphoria or overt emotional turmoil. While we ultimately do not know if there was a specific negative experience precipitating his suicide, his wife did not perceive a downward affective shift in the days preceding his death, nor was she aware of any event that could be perceived as a trigger. In addition, despite the GDS-15 having strong reliability as a depression screening instrument for adults 60 years of age and over, it may have underestimated the severity of depression in our 57 -year-old patient [26].

A fourth distinct feature of our case is the positive family history of completed suicide in the context of terminal illness. There is a substantial body of evidence based on family, twin and adoption studies supporting the notion that genetic factors play a significant role in the predisposition to both suicide attempts and completed suicide [27-29]. Neurobiological abnormalities in the brains of suicide victims, particularly within the serotonergic system, have also been identified and explored through molecular genetics research [30,31]. Previous case reports of suicidal behavior in $\mathrm{AD}$ have lacked this heritability aspect and, thus, we believe our case uniquely represents a possible example of genetic transmission of suicide potential in the context of $\mathrm{AD}$.

At last and perhaps the most distinct, our case demonstrates that cognitive impairment does not necessarily preclude patients from executing detailed suicide plans. On the surface, it may appear that neuropsychological performances at the first and second percentiles would not be compatible with the type of advanced planning and decision making that our patient displayed. We assert, however, that this type of assumption is dangerous. It is important for other clinicians to be aware of this flaw in logic and not assume that patients with moderately advanced dementia are no longer capable of a planned suicide by nature of their disease.

Current literature on suicidal behavior in older adults draws several distinctions between impulsive and planned acts. While our patient was not elderly, we believe this literature provides context for this case. Behavioral research with impulsive suicide attempters reveals impairments in decision making on neuropsychological tests characterized by a lower willingness to delay gratification, also referred to as 'delay discounting' $[21,32,33]$. Impulsive attempters tend to ignore, or discount, the prospect of future rewards and over-focus on negative feedback or past failures, resulting in impulsive decisions [32,34].

Planned and premeditated suicidal behavior, in contrast, has been linked to greater willingness to delay gratification for larger rewards $[21,33]$. Individuals who exhibit highly lethal or planned suicidal behaviors tend to have a higher threshold for acting on suicidal ideation, but demonstrate an inability to flexibly conceptualize their situation and alternative solutions [34]. Thus, impairments in decision making exist across clinical pathways to suicide, but may be differentially expressed.

Recent studies examining variations in brain function in suicide attempters have shed light on the neural circuitry involved. Functional MRI studies implicate abnormalities in basal ganglia and paralimbic activation in poorly planned or impulsive acts, whereas disruptions in the lateral and ventromedial prefrontal cortical activation appear to underlie planned or premeditated acts $[21,32,35]$.

These findings mirror what we have observed clinically in diverse neurodegenerative presentations. We previously reported a case of completed suicide in clinically diagnosed progressive supranuclear palsy, a primarily subcortical dementia syndrome that appeared to be an impulsive, unplanned act [36]. Abnormalities in the subcortical reward circuitry make sense in the context of that particular pathology. Our present case of completed suicide in a clinically diagnosed and autopsy-confirmed cortical dementia syndrome (AD), in contrast, was a much more planned and detailed act, which supports the literature pointing to predominant cortical dysfunction in well-planned versus impulsive acts.

There is some evidence that specific neuropsychological deficits may be associated with suicide potential [33,37,38]. A recent meta-analysis of 25 neuropsychological studies revealed that deficits in decision making, verbal fluency and response inhibition are associated with suicidal behavior in older adults [39]. Our patient, however, demonstrated substantial and diffuse deficits across domains of memory, language, construction and executive functions, with no specific cognitive skill or domain more pronounced or impaired relative to others. Thus, it may be true that certain neuropsychological deficits predispose individuals to risky or suicidal behavior, but not necessarily that those same deficits predict who is likely to complete suicide. 
There is concern within the literature regarding the implications of an early diagnosis of $\mathrm{AD}$ on suicide risk in patients who have relatively intact cognition and awareness early in the course of the disease $[18,40]$. Since the advent of imaging biomarkers for $\mathrm{AD}$ and increased accessibility of amyloid PET scans in recent years, suicide risk in response to positive diagnostic test results has become a major ethical and societal consideration [11]. As we have learned from research with asymptomatic individuals with positive predictive test results for Huntington's disease, serious psychiatric distress and increased suicide risk are plausible consequences [41-43]. Thus, if individuals receive an amyloid PET scan or any of the other emerging biomarker to confirm clinical diagnosis, should screening for depression and suicidal ideation be conducted before and after test results in the interest of risk detection and prevention? We are likely to revisit this question as diagnostic tests for neurodegenerative syndromes are further refined.

At last, one last area of concern is related to genetics and AD. It is worth noting that our patient requested information on genetic testing for $\mathrm{AD}$ prior to his death, but did not complete testing. Thus, although we know the patient's mother developed an unspecified dementia syndrome later in life, we ultimately cannot speak to the possibility of autosomal dominant or familial $\mathrm{AD}$ and whether or not this may impact suicide potential. This is a limitation. Case and cohort studies exploring the psychological impact of predictive and susceptibility testing for AD have thus far failed to identify adverse outcomes [43-47]. Larger, long-term outcome studies, however, are needed.

\section{Future perspective}

Suicide risk prevention is an ever-important topic in healthcare and within communities. For clinicians who treat and evaluate patients with neurodegenerative disorders, safety and risk assessment must become integral components of ongoing care. Exclusively assessing for the presence or absence of depression in this population, however, is not always likely to be sufficient in determining suicide risk. In addition to considering acute depression severity and other overt risk factors, such as a family history of suicidal behavior, we may be able to utilize functional imaging and neuropsychological test results in order to identify individuals with early dementia who may be at elevated risk for suicidal behavior, and thereby intervene before patients act. In addition, advanced cognitive deficits do not preclude the possibility of a planned suicidal act. It is clear from this case how challenging prevention can be despite the best intent of providers and family.

Financial \& competing interests disclosure

MW Haut has received funding from the Conrad N Hilton Foundation; West Virginia Stroke CoBRE (P20 GM109098); the National Institutes of Health (NIH 1R01NS090677-01); and the American College of Radiology. The authors have no other relevant affiliations or financial involvement with any organization or entity with a financial interest in or financial conflict with the subject matter or materials discussed in the manuscript apart from those disclosed.

No writing assistance was utilized in the production of this manuscript.

Ethical conduct of research

The authors state that they have obtained appropriate institutional review board approval or have followed the principles outlined in the Declaration of Helsinki for all human or animal experimental investigations. In addition, for investigations involving human subjects, informed consent has been obtained from the participants involved.

\section{Open access}

This work is licensed under the Attribution-NonCommercial-NoDerivatives 4.0 Unported License. To view a copy of this license, visit http://creativecommons.org/licenses/by-nc-nd/4.0/

\section{References}

1. Folstein MF, Folstein SE, McHugh PR. Mini Mental State: a practical method for grading the cognitive state of patients for clinicians. J. Psychiatr. Res. 12, 189-195 (1975).

2. Frisoni GB, Pievani M, Testa C et al. The topography of grey matter involvement in early and late onset Alzheimer's disease. Brain 130 , 720-730 (2007).

3. Karas G, Scheltens P, Rombouts S et al. Precuneus atrophy in early onset Alzheimer's disease: a morphometric structural MRI study. Neuroradiology 49, 967-976 (2007).

4. Hyman BT, Phelps CH, Beach TG et al. National Institute on Aging-Alzheimer's Association guidelines for the neuropathologic assessment of Alzheimer's disease. Alzheimers Dement. 8, 1-13 (2012). 
5. Schneider B, Maurer K, Frölich L. Dementia and suicide. Fortschr. Neurol. Psychiatr. 69, 164-169 (2001).

6. Grabbe L, Demi A, Camann MA, Potter L. The health status of elderly persons in the last year of life: a comparison of deaths by suicide, injury, and natural causes. Am. J. Public Health 87, 434-437 (1997).

7. Peisah C, Snowden J, Gorrie C, Kril J, Rodriguez M. Investigation of Alzheimer's disease-related pathology in community dwelling older subjects who committed suicide. J. Affect. Disord. 99, 127-132 (2007).

8. Harris EC, Barraclough B. Suicide as an outcome for mental disorders. A meta-analysis. Br. J. Psychiatry 170, 205-228 (1997).

9. Purandare N, Voshaar RCO, Rodway C, Bickley H, Burns A, Kapur N. Suicide in dementia: 9-year national clinical survey in England and Wales. Br. J. Psychiatry 194, 175-180 (2009).

10. Barak Y, Aizenberg D. Suicide among Alzheimer's disease patients: a 10-year survey. Dement. Geriatr. Cogn. Disorder. 14, 101-103 (2002).

11. Draper B, Peisah C, Snowdon J, Brodaty H. Early dementia diagnosis and the risk of suicide and euthanasia. Alzheimers Dement. 6, 75-82 (2010).

12. Serafini G, Calcagno P, Lester D, Girardi P, Amore M, Pompili M. Suicide risk in Alzheimer's disease: a systematic review. Curr. Alzheimer Res. 13, 1083-1099 (2016).

13. Rubio A, Vestner AL, Stewart JM, Forbes NT, Conwell Y, Cox C. Suicide and Alzheimer's pathology in the elderly: a case-controlled study. Biol. Psychiatry 49, 137-145 (2001).

14. Erlangsen A, Zarit SH, Conwell Y. Hospital-diagnosed dementia and suicide: a longitudinal study using prospective, nationwide register data. Am. J. Geriat. Psychiatry 16, 220-228 (2008).

15. Jacobs D, Sano M, Marder K et al. Age at onset of Alzheimer's disease: relation to pattern of cognitive dysfunction and rate of decline. Neurology 44, 1215-1215 (1994).

16. Rhode K, Peskind ER, Raskind MA. Suicide in two patients with Alzheimer's disease. J. Am. Geriatr. Soc. 43, 187-189 (1995).

17. Ferris SH, Hofeldt GT, Carbone G, Masciandaro P, Troetel WM, Imbimbo BP. Suicide in two patients with a diagnosis of probable Alzheimer disease. Alzheimer Dis. Assoc. Disord. 13, 88-90 (1999).

18. Lim WS, Rubin EH, Coats M, Morris JC. Early-stage Alzheimer's disease represents increased suicidal risks in relation to later stages. Alz. Dis. Assoc. Disord. 19, 214-219 (2005).

19. Margo GM, Finkel JA. Early dementia as a risk factor for suicide. Hosp. Community Psychiatry 41, 676-678 (1990).

20. Lesco PA. Murder-suicide in Alzheimer's disease. J. Am. Geriatr. Soc. 37, 167-168 (1989).

21. Vanyukov PM, Szanto K, Hallquist MN et al. Paralimbic and lateral prefrontal encoding of reward value during intertemporal choice in attempted suicide. Psychol. Med. 46, 381-391 (2016).

22. Stern Y. Cognitive reserve in ageing and Alzheimer's disease. Lancet Neurol. 11, 1006-1012 (2012).

23. Van Orden KA, Witte TK, Cukrowicz KC, Braithwaite S, Selby EA, Joiner TE. The interpersonal theory of suicide. Psychol. Rev. 117, 575-600 (2010).

24. Weishaar ME, Beck AT. Hopelessness and suicide. Int. Rev. Psychiatry 4, 177-184 (1992).

25. Beck AT, Kovacs M, Weissman A. Hopelessness and suicidal behavior: an overview. JAMA 234, 1146-1149 (1975).

26. Almeida OP, Almeida SA. Short versions of the Geriatric Depression Scale: a study of their validity for the diagnosis of a major depressive episode according to ICD-10 and DSM-IV. Int. J. Geriat. Psychiatry 14, 858-865 (1999).

27. Roy A, Nielsen D, Rylander G, Sarchiapone M, Segal N. Genetics of suicide in depression. J. Clin. Psychiatry 60, 12-17 (1999).

28. Baldessarini RJ, Hennen J. Genetics of suicide: an overview. Harv. Rev. Psychiatry 12, 1-13 (2004).

29. Brent DA, Mann JJ. Family genetic studies, suicide and suicidal behavior. Am. J. Med. Genet. C. Semin. Med. Genet. 133, 13-24 (2005).

30. Mann JJ. The neurobiology of suicide. Nat. Med. 4, 25-30 (1998).

31. Mann JJ, Currier DM. Stress, genetics and epigenetic effects on the neurobiology of suicidal behavior and depression. Eur. Psychiatry 25, 268-271 (2010).

32. Dombrovski AY, Szanto K, Clark L, Reynolds CF, Siegle GJ. Reward signals, attempted suicide and impulsivity in late-life depression. JAMA Psychiatry 70, 1020-1030 (2013).

33. Dombrovski AY, Hallquist MN. The decision neuroscience perspective on suicidal behavior: evidence and hypotheses. Curr. Opin. Psychiatry 30, 7-14 (2017).

34. Szanto K, de Bruin WB, Parker AM, Hallquist MN, Vanyukov PM, Dombrovski AY. Decision-making competence and attempted suicide. J. Clin. Psychiatry 76, e1590-e1597 (2015).

35. Bartra O, McGuire JT, Kable JW. The valuation system: a coordinate-based meta-analysis of BOLD fMRI experiments examining neural correlates of subjective value. Neuroimage 76, 412-427 (2013).

36. Wiener J, Moran MT, Haut MW. Completed suicide in a case of clinically diagnosed progressive supranuclear palsy. Neurodegener. Dis. Manag. 5, 289-292 (2015). 
37. Kasckow J, Youk A, Anderson SJ et al. Trajectories of suicidal ideation in depressed older adults undergoing antidepressant treatment. J. Psychiatr. Res. 73, 96-101 (2015).

38. Richard-Devantoy S, Szanto K, Butters MA, Kalkus J, Dombrovski AY. Cognitive inhibition in elderly high-lethality suicide attempters. Int. J. Geriatr. Psychiatry 30, 274-283 (2015).

39. Richard-Devantoy S, Berlim MT, Jollant F. A meta-analysis of neuropsychological markers of vulnerability to suicidal behavior in mood disorders. Psychol. Med. 44, 1663-1673 (2014).

40. Mattsson N, Brax D, Zetterberg H. To know or not to know: ethical issues related to early diagnosis of Alzheimer's disease. Int. J. Alzheimers Dis. 2010, 841941 (2010).

41. Di Maio L, Squitieri F, Napolitano G, Campanella G, Trofatter JA, Conneally PM. Suicide risk in Huntington's disease. J. Med. Genet. 30, 293-295 (1993).

42. Almqvist EW, Block M, Brinkman R, Craufurd D, Hayden MR. A worldwide assessment of the frequency of suicide, suicide attempts or psychiatric hospitalization after predictive testing for Huntington's disease. Am. J. Hum. Genet. 64, 1293-1304 (1999).

43. Steinbart EJ, Smith CO, Poorkaj P, Bird TD. Impact of DNA testing for early onset familial Alzheimer disease and frontotemporal dementia. Arch. Neurol. 58, 1828-1831 (2001).

44. Lannfelt L, Axelman K, Lilius L, Basun H. Genetic counseling in a Swedish Alzheimer family with amyloid precursor protein mutation. Am. J. Hum. Genet. 56, 332-335 (1995).

45. Green RC, Roberts JS, Cupples LA et al. Disclosure of APOE genotype for risk of Alzheimer's disease. N. Engl. J. Med. 361, 245-254 (2009).

46. Caselli RJ, Marchant GE, Hunt KS et al. Predictive testing for Alzheiemr's disease: suicidal ideation in healthy participants. Alzheimer Dis Assoc Disord 29, 252-254 (2015).

47. Paulsen JS, Nance M, Kim J et al. A review of quality of life after predictive testing for and earlier identification of neurodegenerative diseases. Prog. Neurobiol. 110, 2-28 (2013). 